\title{
SPP waves in "dielectric-metal-dielectric" structures: influence of exchange correlations
}

\author{
Kostrobij P., Pavlysh V., Nevinskyi D., Polovyi V. \\ Lviv Polytechnic National University \\ 12 S. Bandera str., 79013, Lviv, Ukraine
}

(Received 25 December 2017)

\begin{abstract}
In this paper the results of investigation of the spectrum and the propagation length of SPP waves in the structures synthesized by the authors and the results of mathematical modeling of these characteristics are presented. It is shown that taking into account the simplest (interchange) interactions of metal layer electrons leads to considerable changes in the behaviour of the spectrum of SPP waves and their propagation length.
\end{abstract}

Keywords: SPP wave, DMD structure, 2D electron gas.

2000 MSC: $78 \mathrm{~A} 50,78-05,78 \mathrm{~A} 25$

UDC: $537.5 .8: 535.5: 519.6$

DOI: $10.23939 / \mathrm{mmc} 2017.02 .148$

\section{Introduction}

Surface plasmon-polariton waves (SPP waves) (i.e., electron density undulation) exist in the vicinity of the interface between a dielectric medium and a metal [1-3]. A free electron plasma provides the electromagnetic field confinement near the metal-dielectric interface of metals, making the real part of the metal dielectric permittivity negative in a wide range of frequencies up to the near-ultraviolet domain.

State-of-art approaches to mathematical modeling of propagation of SPP waves are based on the classical Drude theory $[2,3]$ which, as is known $[4,5]$, does not consider spatial dispersion of metal dielectric permittivity $\varepsilon(\boldsymbol{r}, t)$ and effects caused by the interaction of electrons in a metal layer. Those effects become especially significant (and in the same time complicated for modeling) in a low-scale (nanoscale) metallic systems which are used in "dielectric-metal-dielectric" structures (DMD structures) synthesis $[6,7]$.

An attempt of investigation of an electronic correlations influence on SPP waves characteristics for "dielectric-graphene-dielectric" structures was made in [8], where it was shown that the consideration of electronic correlations for 2D-dimensional graphene layers leads to the change of $\omega(\boldsymbol{Q})$ spectrum behaviour of SPP waves even in the case of a small $\left(|\boldsymbol{Q}| \ll k_{F}^{-1}\right)$ wavevector. This confirms (domain $\boldsymbol{Q} \rightarrow 0$ is the domain of the Drude model correctness) the necessity of mathematical modeling describing SPP waves that would take into account anisotropy and electronic correlations effects.

In the paper there is considered the problem of mathematical modeling of SPP waves propagation in a layered DMD structure synthesized by the authors (Pavlysh, Nevinskyi) in the case of modeling the metal layer by two-dimensional electronic plasma [9]. It is shown that taking into account only the exchange correlations leads to considerable changes in the spectrum $\omega(\boldsymbol{Q})$ as well as in the propagation length of SPP wave.

\section{Experiment}

Onto the previously cleared glass $\left(\mathrm{SiO}_{2}\right)$ substrate in a deep vacuum $\left(10^{-8}\right.$ Torr $)$ there was deposited a golden film of the controlled thickness $50 \mathrm{~nm}$. The obtained structure "dielectric-metal" was covered with a polymer (layer thickness $1550 \mathrm{~nm}$ ) and was illuminated (for excitation of SPP wave) by a 
laser with a wavelength of $632.5 \mathrm{~nm}$ and a pulse frequency of $250 \mathrm{fs}$. Schematic representation of the experiment is shown in Fig. 1.

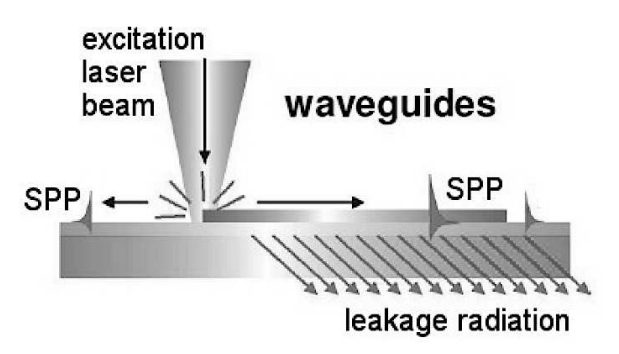

Fig. 1. Schematic representation of SPP excitation.

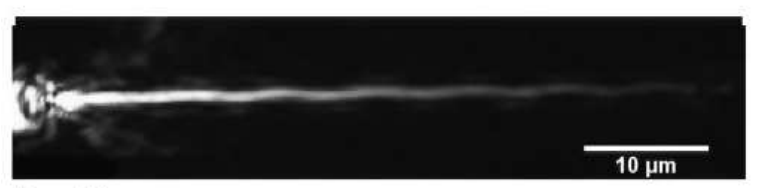

Real Image

Fig. 2 shows the image of SPP wave displayed on LR microscope.

For $150 \mathrm{~nm}$ width of the waveguide, the length of SPP propagation was $10 \mu \mathrm{m}$ and for $200 \mathrm{~nm}-20 \mu \mathrm{m}$. Fig. 3 presents the result of SPP wave measurement depending on the distance to the excitation point.

Such experiments allow us to state that propagation length of SPP wave is $\sim 20 \mu \mathrm{m}$.

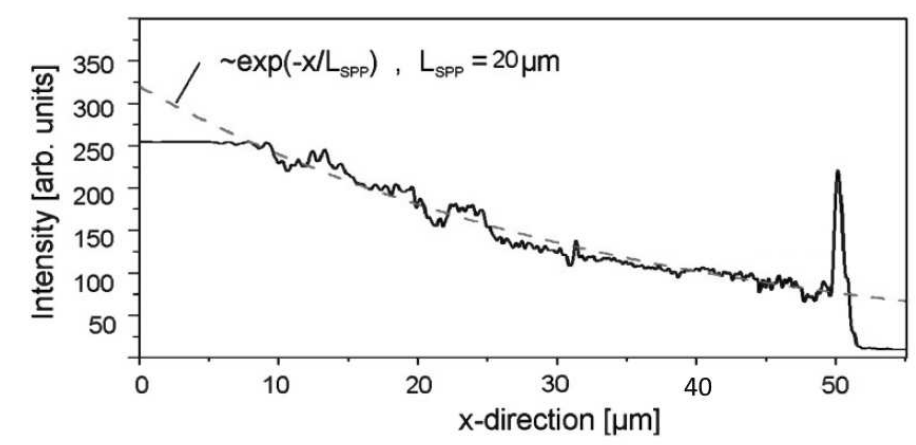

Fig. 3. The intensity of SPP propagation along the waveguide. The solid curve corresponds to the experimental data, the dashed curve - to the theoretical calculation of SPP attenuation.

\section{Mathematical model}

For describing processes of SPP waves emergence and propagation on the surface of nanoscale film covered with dielectrics on top and on bottom ("dielectric-metal-dielectric" structure), we will use the model shown in Fig. 4; $\Omega=\Omega_{1} \cup \Omega_{2} \cup \Omega_{3}$, where $\Omega_{1}=\mathbb{R}^{2} \times(+\delta,+\infty), \Omega_{2}=\mathbb{R}^{2} \times[-\delta,+\delta]$, $\Omega_{3}=\mathbb{R}^{2} \times(-\delta,-\infty)$.

As initial relationships for the construction of mathematical model, we will use the system of Maxwell's equations [4,5]

$$
\begin{gathered}
\operatorname{rot} \boldsymbol{H}(\boldsymbol{r}, t)=\frac{\partial \boldsymbol{D}(\boldsymbol{r}, t)}{\partial t}+\boldsymbol{j}(\boldsymbol{r}, t), \quad \operatorname{rot} \boldsymbol{E}(\boldsymbol{r}, t)=\frac{\partial \boldsymbol{B}(\boldsymbol{r}, t)}{\partial t} \\
\operatorname{div} \boldsymbol{D}(\boldsymbol{r}, t)=\rho(\boldsymbol{r}, t), \quad \operatorname{div} \boldsymbol{B}(\boldsymbol{r}, t)=0
\end{gathered}
$$

where $\boldsymbol{D}(\boldsymbol{r}, t)$ is electric flux density, $\boldsymbol{E}(\boldsymbol{r}, t)$ is electric field, $\boldsymbol{B}(\boldsymbol{r}, t)$ is magnetic flux density, $\boldsymbol{H}(\boldsymbol{r}, t)$ is magnetic field strength, $\rho(\boldsymbol{r}, t)$ is electric charge density, $\boldsymbol{j}(\boldsymbol{r}, t)$ is electric current density, $(x, y, z) \in \mathbb{R}^{3}$, $t \in[0, \infty)$ is time.

We assume that interconnection between the vectors of strength $\boldsymbol{H}$

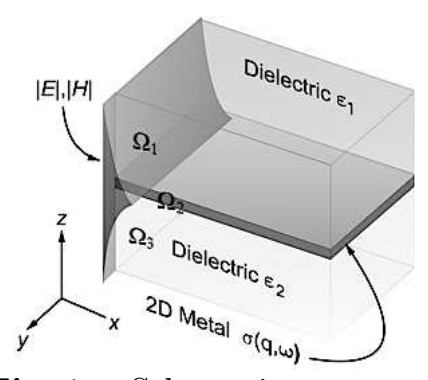

Fig. 4. Schematic representation of "dielectric-metal-dielectric" structure. and $\boldsymbol{E}$ and between $\boldsymbol{B}$ and $\boldsymbol{D}$ electric flux density vectors of electromagnetic field is non-local $[4,5]$, namely

$$
\begin{gathered}
\boldsymbol{D}(\boldsymbol{r}, t)=\int_{\Omega} d \boldsymbol{r}^{\prime} \int_{t} d t^{\prime} \varepsilon\left(\boldsymbol{r}, \boldsymbol{r}^{\prime}, t-t^{\prime}\right) E\left(\boldsymbol{r}^{\prime}, t^{\prime}\right), \\
\boldsymbol{B}(\boldsymbol{r}, t)=\mu_{0} \mu \boldsymbol{H}(\boldsymbol{r}, t), \quad \mu=1,
\end{gathered}
$$

$\mu_{0}$ is magnetic permeability of vacuum, $\varepsilon\left(\boldsymbol{r}, \boldsymbol{r}^{\prime}, t-t^{\prime}\right)$ is the response function (relative permittivity). 
The dependence between current density $\boldsymbol{j}(\boldsymbol{r}, t)$ and electric field vector $\boldsymbol{E}(\boldsymbol{r}, t)$ is similar to (2)

$$
\boldsymbol{j}(\boldsymbol{r}, t)=\int_{\Omega} d \boldsymbol{r}^{\prime} \int_{t} d t^{\prime} \sigma\left(\boldsymbol{r}, \boldsymbol{r}^{\prime}, t-t^{\prime}\right) E\left(\boldsymbol{r}^{\prime}, t^{\prime}\right)
$$

$\sigma\left(\boldsymbol{r}, \boldsymbol{r}^{\prime}, t-t^{\prime}\right)$ is dynamic conductivity.

We will search for a solution of the system (1) for so called TM-waves [2,3], i.e. we assume that

$$
\boldsymbol{H}=\left(0, H_{y}, 0\right), \quad \boldsymbol{E}=\left(E_{x}, 0, E_{z}\right)
$$

For TM-waves $\frac{\partial H_{y}}{\partial y} \equiv 0[2,3]$, therefore $H_{y}=H(x, z, t)$. This means that for our model (2) (it is easy to show) $E_{x}=E_{x}(x, z, t), E_{z}=E_{z}(x, z, t)$, thus the electric field is uniform along $O Y$.

Let us introduce into the model the assumptions about the structure of the functions $\varepsilon\left(\boldsymbol{r}, \boldsymbol{r}^{\prime}, t-t^{\prime}\right)$ and $\sigma\left(\boldsymbol{r}, \boldsymbol{r}^{\prime}, t-t^{\prime}\right)$. We assume that in domains $\Omega_{1}$ and $\Omega_{3}$ (dielectrics)

$$
\varepsilon_{i}\left(\boldsymbol{r}, \boldsymbol{r}^{\prime}, t-t^{\prime}\right) \cong \varepsilon_{0} \varepsilon_{i}\left(t-t^{\prime}\right) \delta\left(\boldsymbol{r}-\boldsymbol{r}^{\prime}\right), \quad i=1,3,
$$

where $\varepsilon_{0}$ is the vacuum permittivity. This means that in the dielectric environment we will neglect the spatial dispersion and will take into account only the frequency dispersion $[4,5]$. Since in dielectrics free carriers of charge are absent we will assume that in this domains $\sigma\left(\boldsymbol{r}, \boldsymbol{r}^{\prime}, t-t^{\prime}\right) \equiv 0$.

For describing the electromagnetic field behavior in the domain $\Omega_{2}$ (metal nanoscale film), the functions $\varepsilon\left(\boldsymbol{r}, \boldsymbol{r}^{\prime}, t-t^{\prime}\right)$ and $\sigma\left(\boldsymbol{r}, \boldsymbol{r}^{\prime}, t-t^{\prime}\right)$ we will model by functions of 2D-electron liquid [8,9], namely, we will assume that

$$
\begin{aligned}
& \varepsilon_{2}\left(\boldsymbol{r}, \boldsymbol{r}^{\prime}, t-t^{\prime}\right) \cong \varepsilon_{0} \varepsilon_{2}\left(\boldsymbol{r}_{\|}-\boldsymbol{r}_{\|}^{\prime}, t-t^{\prime}\right) \delta\left(z-z^{\prime}\right), \\
& \sigma_{2}\left(\boldsymbol{r}, \boldsymbol{r}^{\prime}, t-t^{\prime}\right) \cong \sigma_{0} \sigma_{2}\left(\boldsymbol{r}_{\|}-\boldsymbol{r}_{\|}^{\prime}, t-t^{\prime}\right) \delta\left(z-z^{\prime}\right),
\end{aligned}
$$

$\boldsymbol{r}_{\|}$is a component of the radius vector $\boldsymbol{r}$ that lies in $X O Y$, i.e. $\boldsymbol{r}=\left(\boldsymbol{r}_{\|}, z\right)$.

Under such assumptions we are neglecting the dispersion $\varepsilon_{2}$ and $\sigma_{2}$ along $O Z$, which is, generally speaking, true for metal films with the thickness $2 \delta$ that satisfies the condition $2 \delta k_{F} \sim 1 \div 10$, where $k_{F}=\left(\frac{3}{4 \pi}\right)^{\frac{2}{3}} r_{s} a_{0}^{-1}, r_{s}$ is Gell-Mann-Brakner parameter, $a_{0}$ is Bohr raduis $[6,7]$ and the influence of fringe effects in the $X O Y$ plane (this is true when geometric dimensions of a metal film in $X O Y$ are considerably larger than its thickness along $O Z, 2 \delta(\sqrt{S})^{-1} \ll 1$, where $S$ is an area of the metal film surface).

For the further analysis of the system of Maxwell's equations it is convenient to write it down for Fourier components of $\boldsymbol{H}, \boldsymbol{E}, \boldsymbol{B}, \boldsymbol{D}$ vectors (all the characteristics of the model is homogeneous with respect to time), by defining the Fourier transform with respect to time as [10]

$$
f(t)=\frac{1}{2 \pi} \int_{-\infty}^{\infty} \tilde{f}(\omega) e^{i \omega t} d \omega, \quad \tilde{f}(\omega)=\frac{1}{2 \pi} \int_{-\infty}^{\infty} f(t) e^{-i \omega t} d \omega
$$

we have:

- domain $\Omega_{1}$ :

$$
\operatorname{rot} \boldsymbol{H}(\boldsymbol{r}, \omega)=i \omega \varepsilon_{0} \tilde{\varepsilon}_{1}(\omega) \boldsymbol{E}(\boldsymbol{r}, \omega)
$$

- domain $\Omega_{3}$ :

$$
\operatorname{rot} \boldsymbol{H}(\boldsymbol{r}, \omega)=i \omega \varepsilon_{0} \tilde{\varepsilon}_{3}(\omega) \boldsymbol{E}(\boldsymbol{r}, \omega)
$$

- domain $\Omega_{2}$ :

$$
\operatorname{rot} \boldsymbol{H}(\boldsymbol{r}, \omega)=i \omega \varepsilon_{0} \int_{\Omega_{2}} d \boldsymbol{r}_{\|}^{\prime} \tilde{\varepsilon}_{2}\left(\boldsymbol{r}_{\|}-\boldsymbol{r}_{\|}^{\prime}, \omega\right) \boldsymbol{E}\left(\boldsymbol{r}_{\|}^{\prime}, \omega, z\right)+\sigma_{0} \int_{\Omega_{2}} d \boldsymbol{r}_{\|}^{\prime} \tilde{\sigma}_{2}\left(\boldsymbol{r}_{\|}-\boldsymbol{r}_{\|}^{\prime}, \omega\right) \boldsymbol{E}\left(\boldsymbol{r}_{\|}^{\prime}, \omega, z\right) .
$$


For the domain $\Omega_{2}(9)$ it is also convenient to define the Fourier transform with respect to $\boldsymbol{r}_{\|}$that allows us to write (9) as follows:

$$
\operatorname{rot} \boldsymbol{H}\left(\boldsymbol{r}_{\|}, \omega\right)=\frac{i \omega \varepsilon_{0}}{(2 \pi)^{2}} \int_{\Omega_{2}} d \tilde{\boldsymbol{q}} \tilde{\varepsilon_{2}}(\boldsymbol{q}, \omega) e^{i\left(\boldsymbol{q}, \boldsymbol{r}_{\|}^{\prime}\right)} \boldsymbol{E}(\boldsymbol{q}, \omega, z)+\frac{\sigma_{0}}{(2 \pi)^{2}} \int_{\Omega_{2}} d \boldsymbol{q} \tilde{\sigma_{2}}(\boldsymbol{q}, \omega) e^{i\left(\boldsymbol{q}, \boldsymbol{r}_{\|}^{\prime}\right)} \boldsymbol{E}(\boldsymbol{q}, \omega, z),
$$

where $\boldsymbol{q}=\left(q_{x}, q_{y}\right), d \boldsymbol{q}=\left(d q_{x}, d q_{y}\right),\left(\boldsymbol{q}, \boldsymbol{r}_{\|}\right) \equiv q_{x} x+q_{y} y$.

For describing of SPP waves propagation we will search solutions of the system (7)-(10) in the form of the following "ansatz"

$$
H_{y}(x, \omega, z)=H_{y}(z) e^{i Q x} .
$$

It is obviuos that in this case

$$
\boldsymbol{E}(\boldsymbol{r}, \omega)=\boldsymbol{E}(x, \omega, z)=\boldsymbol{E}(\omega, z) e^{i Q x} .
$$

Since for such "ansatz"

$$
\boldsymbol{E}(\boldsymbol{q}, \omega, z)=(2 \pi)^{2} \boldsymbol{E}(\omega, z) \delta\left(Q-q_{x}\right) \delta\left(-q_{y}\right),
$$

$(\delta(\ldots)$ is the Dirac delta function [10]), then the system of equations (7)-(10) takes the form

$$
\begin{gathered}
\operatorname{rot} \boldsymbol{H}(Q, \omega, z)=i \omega \varepsilon_{0} \tilde{\varepsilon}_{2}(\omega) \boldsymbol{E}(Q, \omega, z), \quad j=1,3, \\
\operatorname{rot} \boldsymbol{H}(Q, \omega, z)=i \omega \varepsilon_{0} \tilde{\varepsilon}_{2}(Q, \omega) \boldsymbol{E}(Q, \omega, z)+\tilde{\sigma}(Q, \omega) \boldsymbol{E}(Q, \omega, z) .
\end{gathered}
$$

Complementing the system (13), (14) with the Helmholtz equations [5] for $\boldsymbol{H}(\omega, z)$ (which are easy to obtain for such a layered structure) we have a mathematical model of SPP waves propagation:

- domain $\Omega_{1}$ :

$$
\begin{gathered}
\frac{d^{2} H_{y}(\omega, z)}{d z^{2}}-\left(Q^{2}-\tilde{\varepsilon}_{1}(\omega) \frac{\omega^{2}}{c^{2}}\right) H_{y}(\omega, z)=0 \\
E_{x}(\omega, z)=-\frac{i}{\omega \varepsilon_{0} \tilde{\varepsilon}_{1}(\omega)} \frac{\partial H_{y}(\omega, z)}{\partial z} \\
E_{z}(\omega, z)=\frac{Q}{\omega \varepsilon_{0} \tilde{\varepsilon}_{1}(\omega)} H_{y}(\omega, z)
\end{gathered}
$$

- domain $\Omega_{3}$ :

$$
\begin{gathered}
\frac{d^{2} H_{y}(\omega, z)}{d z^{2}}-\left(Q^{2}-\tilde{\varepsilon}_{3}(\omega) \frac{\omega^{2}}{c^{2}}\right) H_{y}(\omega, z)=0, \\
E_{x}(\omega, z)=-\frac{i}{\omega \varepsilon_{0} \tilde{\varepsilon}_{3}(\omega)} \frac{\partial H_{y}(\omega, z)}{\partial z} \\
E_{z}(\omega, z)=\frac{Q}{\omega \varepsilon_{0} \tilde{\varepsilon}_{3}(\omega)} H_{y}(\omega, z)
\end{gathered}
$$

- domain $\Omega_{2}$ :

$$
\begin{gathered}
\frac{d^{2} H_{y}(\omega, z)}{d z^{2}}-\left(Q^{2}-\gamma(Q, \omega, 0) \frac{\omega^{2}}{c^{2}}\right) H_{y}(\omega, z)=0, \\
-\frac{\partial H_{y}(\omega, z)}{\partial z}=E_{x}(\omega, z)\left(i \omega \varepsilon_{0} \tilde{\varepsilon}_{2}(Q, \omega, 0)-\sigma_{0} \tilde{\sigma}_{2}(Q, \omega, 0)\right), \\
H_{y}(\omega, z)=E_{z}(\omega, z)\left(i \omega \varepsilon_{0} \tilde{\varepsilon}_{2}(Q, \omega, 0)-\sigma_{0} \tilde{\sigma}_{2}(Q, \omega, 0)\right) .
\end{gathered}
$$

The system of equations (15)-(17) should be solved using the corresponding electrodynamic joining conditions at the boundary of environments: continuity of the corresponding components of $\boldsymbol{H}$ and $\boldsymbol{D}$ 
vectors. For the considered in this paper an ultrathin metal film $2 \delta k_{F} \sim 1 \div 10$, we obtain that:

$$
\begin{gathered}
H_{y}^{(1)}(\omega, z)=A_{1} e^{-\lambda z}, \quad E_{x}^{(1)}(\omega, z)=\frac{\lambda}{\omega \varepsilon_{0} \tilde{\varepsilon}_{1}(\omega)} A_{1} e^{-\lambda z}, \quad E_{z}^{(1)}(\omega, z)=\frac{Q}{\omega \varepsilon_{0} \tilde{\varepsilon}_{1}(\omega)} A_{1} e^{-\lambda z} \\
\lambda=\sqrt{Q^{2}+\frac{\omega^{2}}{c^{2}} \tilde{\varepsilon}_{1}(\omega)}, \\
H_{y}^{(3)}(\omega, z)=A_{1} e^{\xi z}, \quad E_{x}^{(3)}(\omega, z)=\frac{\lambda}{\omega \varepsilon_{0} \tilde{\varepsilon}_{3}(\omega)} B_{3} e^{\xi z}, \quad E_{z}^{(3)}(\omega, z)=\frac{Q}{\omega \varepsilon_{0} \tilde{\varepsilon}_{3}(\omega)} B_{3} e^{\xi z}, \\
\xi=\sqrt{Q^{2}+\frac{\omega^{2}}{c^{2}} \tilde{\varepsilon}_{3}(\omega)}, \\
c^{2}=\varepsilon_{0} \mu_{0} .
\end{gathered}
$$

The constants $A_{1}$ and $B_{3}$ we will obtain from the joining conditions of the solutions (18), (19) at the boundary $z=0$, taking into account that at this boundary there exists the surface current $j_{x}=\sigma_{0} \sigma(Q, \omega) \tilde{E}_{x}(Q, \omega, 0)$, where $\tilde{E}_{x}$ is electric field in the plane $z=0$. From this condition it follows that

$$
\begin{gathered}
H_{y}^{(1)}(\omega,+0)-H_{y}^{(3)}(\omega,-0)=j_{x}, \\
\varepsilon_{0} \tilde{\varepsilon}_{1}(\omega) E_{y}^{(1)}(\omega,+0)=\varepsilon_{0} \tilde{\varepsilon}_{3}(\omega) E_{y}^{(3)}(\omega,-0)=\rho_{x}, \\
E_{z}^{(1)}(\omega,+0)=E_{z}^{(3)}(\omega,-0) .
\end{gathered}
$$

Non-zero solution of the equation system (20) exists only if the following condition is fulfilled

$$
\frac{\tilde{\varepsilon}_{1}(\omega)}{\lambda}+\frac{\tilde{\varepsilon}_{3}(\omega)}{\xi}=-\frac{1}{\omega \varepsilon_{0}} \sigma(Q, \omega)
$$

which is dispersion relation for finding $\omega(Q)$ of SPP wave in this model. Notice that the dispersion equation (21) coincides with the dispersion equation of the SPP wave spectrum obtained in [8] for the case of "dielectric-graphene-dielectric" model.

\section{Solution of dispersion equation}

To solve the equation (21), a two-dimensional dynamic conductivity of an electron liquid $\sigma(Q, \omega)$ should be set. In the paper, solutions of (21) for two well-known in literature models of $\sigma(Q, \omega)$ are investigated:

- the Drude model [2]:

$$
\sigma_{0} \sigma(Q, \omega)=\frac{e^{2} \varepsilon_{F}}{\pi \hbar^{2}} \frac{i}{\omega+i \tau^{-1}}
$$

- the RPA model [9]:

$$
\begin{gathered}
\sigma(Q, \omega)=-i \omega \chi(Q, \omega), \quad \chi(Q, \omega)=\chi_{1}(Q, \omega)+i \chi_{2}(Q, \omega), \\
\chi_{1}=G\left(\frac{Q}{k_{F}}-C_{-}\left(\left(\frac{Q}{2 k_{F}}-\frac{\omega}{Q v_{F}}\right)^{2}-1\right)^{\frac{1}{2}}-C_{+}\left(\left(\frac{Q}{2 k_{F}}+\frac{\omega}{Q v_{F}}\right)^{2}-1\right)^{\frac{1}{2}}\right), \\
\chi_{2}=G\left(D_{-}\left(1-\left(\frac{Q}{2 k_{F}}-\frac{\omega}{Q v_{F}}\right)^{2}\right)^{\frac{1}{2}}-D_{+}\left(1-\left(\frac{Q}{2 k_{F}}+\frac{\omega}{Q v_{F}}\right)^{2}\right)^{\frac{1}{2}}\right), \\
G=\frac{N e^{2}}{\varepsilon_{0} m q^{2} v_{F}^{2}}, \quad v_{F}=\frac{\hbar k_{F}}{m},
\end{gathered}
$$




$$
\begin{gathered}
C_{ \pm}=\frac{\left(\frac{Q}{2 k_{F}} \pm \frac{\omega}{Q v_{F}}\right)}{\left|\frac{Q}{2 k_{F}}+\frac{\omega}{Q v_{F}}\right|}, \quad D_{ \pm}=0 \text { for }\left|\frac{Q}{2 k_{F}}+\frac{\omega}{Q v_{F}}\right|>1, \\
C_{ \pm}=0, \quad D_{ \pm}=1 \text { for }\left|\frac{Q}{2 k_{F}}+\frac{\omega}{Q v_{F}}\right|<1
\end{gathered}
$$

In the given expressions (22)-(24) for dynamic conductivity $k_{F}$ is the Fermi momentum, $\varepsilon_{F}=\frac{\hbar^{2} k_{F}^{2}}{2 m}$ is the Fermi energy, $v_{F}$ is the Fermi velocity, $\hbar$ is reduced Plank constant, $m, e$ are the mass and the charge of electron respectively (see [11]), $\tau^{-1}$ is the reverse dumping time [8], $\tau=1.3 \cdot 10^{-13}$ [3].

Numerical analysis of the equation was carried out using "MATLAB" for layered structure "polyethylene-gold- $\mathrm{SiO}_{2}$ " with parameters:

$$
\varepsilon_{1}(\omega)=\tilde{\varepsilon}_{1}(+\infty)=2.3, \quad \varepsilon_{3}(\omega)=\tilde{\varepsilon}_{3}(-\infty)=4, \quad k_{F} a_{0}=\left(\frac{3}{4 \pi}\right)^{\frac{2}{3}} r_{s}, \quad r_{s}=2.9 .
$$

The results of numerical calculation of $\omega=\omega(Q)$ are presented in Figs. 5-7.
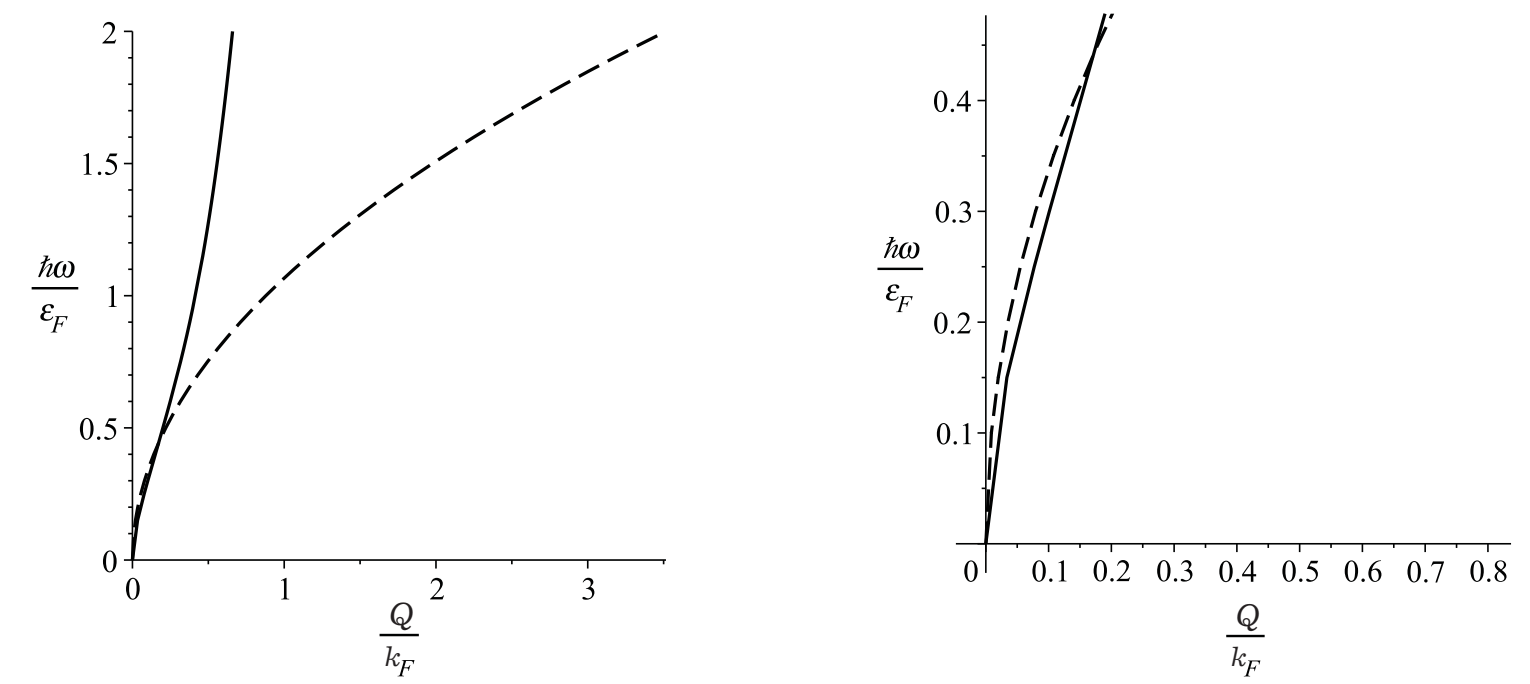

Fig. 5. Graphics of dispersion $\omega(Q)$ for the Drude model (dashed curves) and for the RPA model (solid curves).

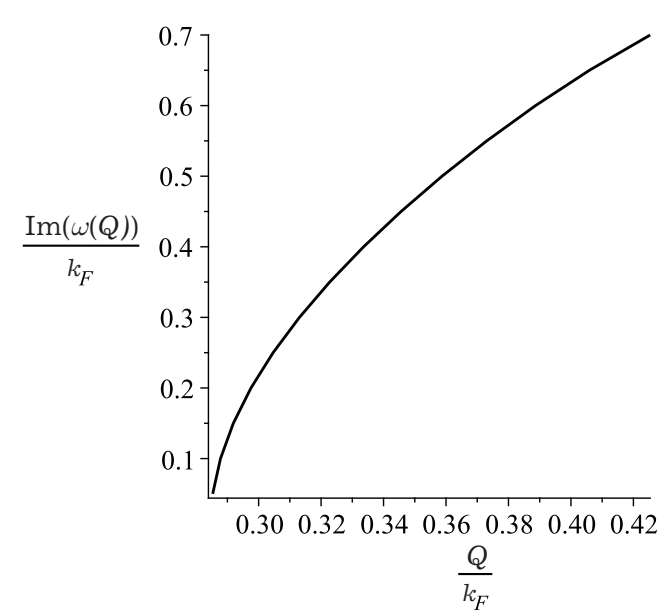

Fig. 6. Imaginary part $\operatorname{Im}(\omega(Q))$ of disperse relation solution for the Drude model.

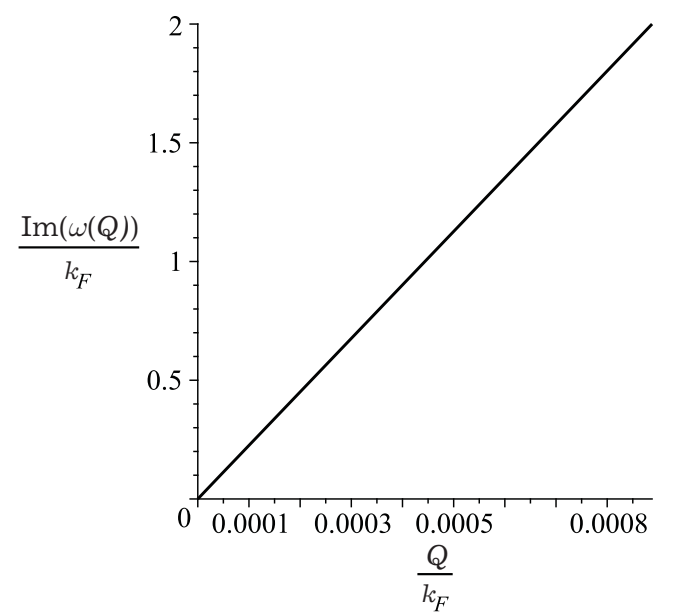

Fig. 7. Imaginary part $\operatorname{Im}(\omega(Q))$ of disperse relation solution for the RPA model. 
As we can see in Fig. 5, the difference between the Drude and the RPA models becomes considerable when the wavevector $Q$ in the domain is $\frac{|Q|}{k_{F}} \sim 0.05$. This means that for describing $\varepsilon(\boldsymbol{r}, t)$ and $\sigma(\boldsymbol{r}, t)$ other models should be used instead of the Drude model. A similar result was obtained for "dielectricgraphene-dielectric" structures [8].

Figs. 6, 7 present the dependence of the imaginary part of wavevector $\frac{\operatorname{Im}(Q)}{k_{F}}$ on $\frac{Q}{k_{F}}$. This characteristic can be interpreted as inverse propagation length of $\operatorname{SPP}\left(L_{S P P}^{-1}\right)$. For the Drude model $L_{S P P} \sim 0.2 \mu \mathrm{m}$ and for the RPA model $L_{S P P} \sim 0.003 \mu \mathrm{m}$. Decreasing of propagation length for the RPA model is understandable because this model takes into account the plasmon scattering effects connected with interchange interactions (Landau damping) [8]. The propagation length $L_{S P P} \sim 20 \mu \mathrm{m}$ is obtained experimentally due to a laser illumination.

\section{Conclusions}

Being obtained as a result of mathematical modeling the dependences of the spectrum $\omega(Q)$ and the propagation length $L_{S P P}$ of the plasmon-polariton wave show that for theoretical investigation of these characteristics we should take into account both interaction effects of electrons in a metal layer and anisotropy effects of a metal film in DMD structures. This will be a subject of the future researches.

[1] Kaminov I. P., Mammel W. L., Weber H. P. Metal-Clad Optical Waveguides: Analytical and Experimental Study. Appl. Opt. 13 (2), 396-405 (1974).

[2] Maier S. A. Plasmonics: Fundamentals and Application. Springer-Verlag (2007).

[3] Brongersma M. I., Kik P. G. Surface Plasmons Nanophotonics. Springer-Verlag (2007).

[4] Landau L. D., Bell J. S., Kearsley M. J, Pitaevskii L. P, Lifshitz E. M., Sykes J. B. Electrodynamics of continuous media. Pergamon Press, Vol. 8 (1984).

[5] Jackson J. D. Classical Electrodynamics. John Wiley \& Sons (2007).

[6] Kurbatsky V.P. Dielectric tensor of low-dimensional metal systems. J. Exp. Theor. Phys. 125 (1), 148-158 (2017).

[7] Wood D. M., Ashcroft N. W. Quantum size effects in the optical properties of small metallic particles. Phys. Rev. B. 25 (10), 6255-6274 (1982).

[8] Jablan M., Buljan H., Soljačić M. Plasmonics in graphene at infrared frequencies. Phys. Rev. B. 80 (24), 245435 (2009).

[9] Ando T., Fowler A. B., Stern F. Electronic properties of two-dimensional systems. Rev. Mod. Phys. 54 (2), 437-672 (1982).

[10] Svidzyns'kyy A. V. Matematychni metody teoretychnoyi fizyky. Kyiv, ITF im. M. M. Boholyubova NAN Ukrayiny (2009), (in Ukrainian).

[11] Vakarchuk I. O. Kvantova mekhanika. Lviv, LNU im. I. Franka (2007), (in Ukrainian). 


\title{
SPP-хвилі в структурах «діелетрик-метал-діелектрик»: вплив обмінних кореляцій
}

\author{
Костробій П., Павлиш В., Невінський Д., Польовий В. \\ Національний університет «Львівсъка політехніка» \\ вул. С. Бандери, 12, 79013, Львів, Украӥна
}

\begin{abstract}
Подано результати дослідження спектра та довжини поширення SPP-хвиль в синтезованих авторами структурах та результати математичного моделювання цих характеристик. Показано, що врахування найпростіших (обмінних) взаємодій електронів металевого прошарку приводить до значних змін у поведінці спектра SPP-хвиль та довжини їхнього поширення.
\end{abstract}

Ключові слова: SPP-хвилі, ДМД-структури, електроний $2 D$-газ.

2000 MSC: 78A50, 78-05, 78A25

удк: 537.5.8:535.5:519.6 